\title{
Scanning Probe Microscopy of Silicone Treated Cellulose Fibers: A Comparison of In-Situ, LFM, and Phase Imaging for Characterization of Adsorbed vs. Covalently Bonded Silicone Coatings
}

\author{
Julie Teetsov, ${ }^{*}$ Matthew Butts, Steven Stoessel, Kathryn Shaffer, and Mike Burrell
}

General Electric Global Research Center, 1 Research Circle, Niskayuna, NY 12309, U.S.A.

There is added value in designing experiments that probe the nanostructure-property relationships of coatings in the environment that most closely mimics their end-use [1]. We use scanning probe microscopy to image cellulose fibers treated with two types of poly(dimethylsiloxane) (PDMS) polymers, $\mathbf{1}$ and $\mathbf{2}$, designed to provide adsorptive versus covalent binding to the cellulose, respectively. While 1 is a standard PDMS, 2 has been functionalized to facilitate covalent binding to the fabric surface and improve overall performance. In order to better understand the relationship between silicone-fiber interactions and bulk fabric performance, it was of great importance to characterize differences in surface nanostructure, in the dry and wet state, of different silicone coatings. The nature of the siliconefiber interactions on the molecular scale can then be compared to macroscopic properties. Our experiment compares the topography of treated and untreated cellulose before, during, and after "wetting" with water. With in-situ water imaging, we found that fibers treated with 1 adsorbed to the cellulose rearrange on the surface to form balls (Fig. 1a) in an aqueous environment while fibers treated with 2 forms covalent bonds to the fiber and do not show balls (Fig. 1b) but instead look identical to untreated cellulose fiber surfaces. The nanoresolution of SPM enables in-situ force-distance curve measurements that show a greater visco-elasticity of the balls compared to the background cellulose. We also use phase and lateral force microscopy (LFM) to reveal differences in surface frictional, adhesive, and visco-elastic properties between untreated and treated fibers and between these same fibers before and after wetting. For example, an untreated cellulose fiber is compared to one treated with $\mathbf{2}$ using intermittent mode phase imaging (in air) in Figs. 1c and d, respectively. While both topography images are identical (not shown), the phase image reveals greater phase lag for the treated fiber. This is consistent with the fact that silicone should have greater visco-elasticity than cellulose and increased adhesive forces in air acting on the tip. The opposite effect will take place if silicone acts to reduce tip-sample frictional forces. Therefore phase interpretation is highly qualitative. Figs. 1e and $1 \mathrm{f}$ show the topography and corresponding phase image of a fiber treated with a covalently bound coating taken in air after a wetting treatment. By comparing the phase images before (Fig. 1d) versus after (Fig. 1f) wetting, one can study differences in silicone spreading in treated cellulose as compared to untreated cellulose which displayed no differences before or after wetting. We show SPM to be a unique characterization tool for measuring topography, adhesion, friction, and other mechanical properties of surfaces under both ambient and liquid conditions. The experimental factors leading to variability in LFM and phase imaging along with a comparison of the contrast mechanisms achieved in these two techniques will be discussed.

References:

[1] G. Y. Liu et al., Langmuir. 13 (1997) 343.

[2] J.A.T thanks the application scientists at Digital Instruments for many useful discussions concerning in-situ and LFM imaging.

${ }^{*}$ To whom correspondence should be addressed: 518-387-7551; teetsov@crd.ge.com 

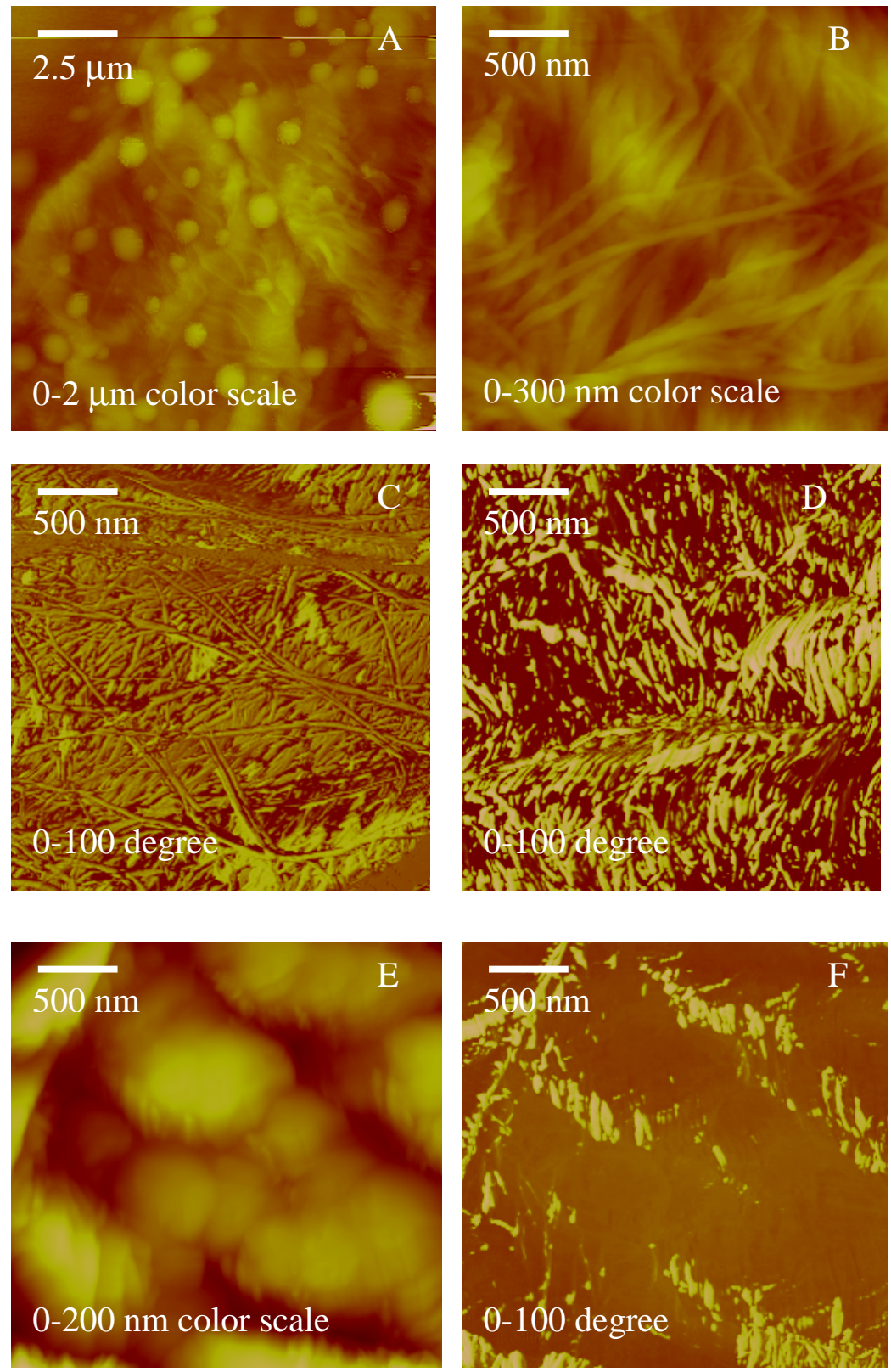

Fig. 1. (a,b) Topography images of cellulose treated with $\mathbf{1}$ and cellulose treated with $\mathbf{2}$ respectively; taken in water, $\mathrm{pH}=7$.

$(\mathrm{c}, \mathrm{d})$ Intermittent mode phase images taken in air of untreated cellulose and cellulose treated with 2 , respectively.

$(\mathrm{e}, \mathrm{f}) \quad$ Topography and phase images, respectively, of cellulose treated with $\mathbf{2}$ taken in air following a wetting treatment.

All images taken at $\mathrm{A}_{\mathrm{sp}}=0.93 \mathrm{~A}_{0}$ tapping force. 